To the Editors:

\title{
Metformin in diabetes mellitus: are we choosing the right patients?
}

Ceylon Medical Journal, 2000; 45: 43

We studied the pattern of metformin use in patients with type 2 diabetes mellitus at the National Hospital of Sri Lanka (NHSL) to identify patients at risk of lactic acidosis in a descriptive, cross-sectional study.

The study was conducted at the diabetic clinic, medical and surgical wards, intensive care units and the cardiology unit of the NHSL in the month of October 1996.

Prescriptions issued from the diabetic clinic for patients with type 2 diabetes (644 prescriptions) were collected from the outdoor dispensary on two consecutive days. Information on drug treatment of patients with type 2 diabetes was obtained from 14 medical wards (91 patients), 14 surgical wards ( 83 patients), the intensive care units (none), and 2 wards of the cardiology unit (13 patients). The patients were interviewed and necessary data were collected from the case notes. The number of metformin tablets issued from 1992 to 1996 was obtained from the Medical Supplies Division (MSD).

Of 644 prescriptions at the diabetic clinic, $310(48 \%)$ had metformin either alone or on combination with other oral hypoglycaemic drugs. $13(4.2 \%)$ prescriptions were for patients aged 70 years and above, and $72(23.2 \%)$ were prescribed $3 \mathrm{~g}$ of metformin daily.

Of the 1547 ward patients studied in the NHSL, 187 were diabetic (12\%) and $31(16.6 \%)$ were taking metformin. Seven patients $(22.6 \%)$ were above 70 years of age and only one patient was taking $3 \mathrm{~g}$ daily of metformin. Nine patients (29\%) taking metformin had contraindications for its use ( 3 renal failure, 3 liver failure and 3 heart failure.)
The numbers of metformin tablets issued by MSD over 5 years are 4.2 million in 1992, 8.1 million in 1993, 9.45 million in 1994, 12.22 million in 1995 and 13.09 million in 1996.

We found that metformin was used in patients with contraindications and those over 70 years of age. $21 \%$ of patients were on $3 \mathrm{~g}$ of metformin daily. The use of metformin is increasing in Sri Lanka. Because of the significant mortality associated with lactic acidosis, prescribers should be aware of contraindications and precautions for the use of metformin.

There were 15 reports of lactic acidosis with the use of metformin reported to the Australian Adverse Drug Reaction Advisory Committee (ADRAC) in 1995 (1). Their ages ranged from 59 to 88 years. In 4 of the 6 fatal cases, the dose of metformin was $3 \mathrm{~g}$ daily. In 9 cases renal failure was noted. All the recently reported fatal cases had contraindications to metformin use (2).

\section{Acknowledgments}

The authors thank Dr. K Withanaarachchi and Dr. S Sri Ranganathan for helping to interview the patients.

\section{References}

1. Fatal lactic acidosis with metformin. Australian Adverse Drug Reaction Bulletin 1995; 14: 6.

2. Stephen N, Davis, Daryl K Granner. Insulin, oral hypoglycaemic agents, pharmacology of the endocrine pancreas. In: Goodman and Gilman's The Pharmacological Basis of Therapeutics. New York: McGraw-Hill; 1996.

K De Abrew, P Galappatthy and K Weerasuriya, Department of Pharmacology, Faculty of Medicine, University of Colombo. 